\title{
DYNAMIC NETWORK TARIFFS: ARE THEY THE MOST EFFICIENT WAY TO MATCH PEAK CONSUMPTION AND NETWORK INCREMENTAL COSTS?
}

\author{
Guido PIRES \\ EDP Distribuição - Portugal \\ guido.pires@edp.pt \\ Ruben Barbosa PINTO \\ INESC-TEC Portugal \\ rbpinto@inesctec.pt
}

\author{
João Tomé SARAIVA \\ INESC-TEC Portugal \\ jsaraiva@fe.up.pt \\ José Nuno FIDALGO \\ INESC-TEC Portugal \\ jfidalgo@fe.up.pt
}

\author{
João Filipe NUNES \\ EDP Distribuição - Portugal \\ joaofilipe.nunes@edp.pt
}

\begin{abstract}
The purpose of this paper is to present the main results of the ongoing analysis of applying dynamic network access tariffs in Portugal.

For the 2015 - 2017 regulatory period, the Portuguese National Regulatory Authority, ERSE, required the three main Portuguese DSOs to submit, until the end of June 2016, plans for the implementation of network dynamic tariff schemes targeting Medium, High and Extra High Voltage customers, as well as the respective cost-benefit analysis. EDP Distribuição, the main Portuguese DSO, is preparing a report regarding the implementation of pilot projects on a sample of these segments of customers, which are due to be on the field during 2017. These pilots should help electrical energy stakeholders understand how the Electric System can benefit from the use of dynamic tariffs focused on networks, allowing for the quantification of benefits in a more accurate way. The level of demand response that results from price signals is a key issue that both the regulator and EDP Distribuição will quantify. Other important issue to assess in this study is the efficiency of cost recovery under a dynamic tariff scheme. In conclusion, this paper will present some results obtained from the cost-benefit analysis regarding the implementation of a Critical Peak Pricing scheme, as well as the key learnings supporting the introduction of dynamic schemes in the future, not only for $E H V, H V$ and $M V$ customers but also eventually extending it to LV ones.
\end{abstract}

\section{INTRODUCTION}

In the present context of the Electric System transformation towards decentralized generation and sustainability, tariff structures face enormous challenges. The rapid growth of renewable injection in the distribution network requires new mechanisms of demand response. Dynamic tariffs may constitute an important means to promote it.

Current tariff schemes in Portugal with a price differentiation establish a static annual number of peak hours. However, peak consumption is strongly related to volatile variables like temperature. Hence, it is only possible to estimate with accuracy real peak consumption few hours / days in advance. The 15-minute analysis of network energy flows, in the last years, revealed a significant mismatch between the peak hours set in the static Time of Use tariffs and the periods of highest network congestion. The implementation of a dynamic scheme could allow for a better correspondence between network incremental costs and peak consumption, which is one of the most relevant drivers of network investment, operation and maintenance costs.

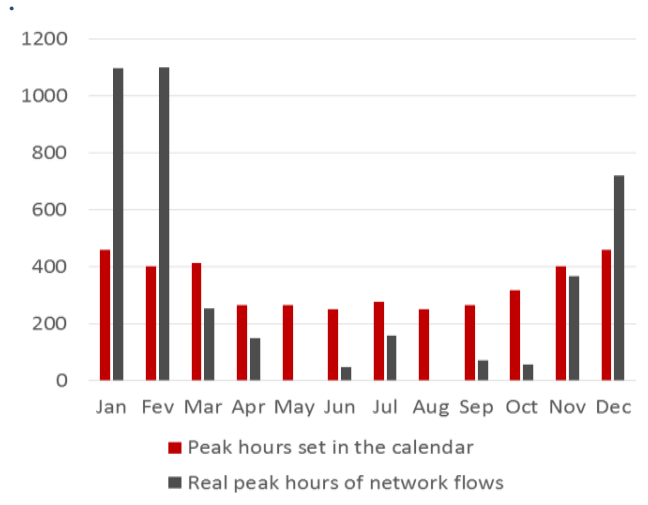

Figure 1 - calendar peak hours vs peak hours of consumption (2014)

In this scope, in December 2014 the Portuguese regulator required EDP Distribuição and the DSOs from Azores and Madeira, the development of a plan for the deployment of pilots regarding the implementation of dynamic network access tariffs in VHV, HV and MV customers. The rational of choice of these segments was not only economical - they have remote metering installed which makes the implementation of dynamic tariff options faster and cheaper - but also because they represent $46 \%$ of total consumption and are more familiar with complex tariff structures.

This requirement led EDP Distribuição to establish a contract with INESC-TEC, which would support the DSO in the cost-benefit analysis and the plan for the pilot. 
Regarding the latter, many parameters had to be selected: the tariff scheme, the number of critical periods and their duration, the timing to notify the customers, the price levels and the criteria for critical period activation.

One of the most important challenges in this work has been the identification of the major benefits of such schemes, which depend on the level of consumer response and are not usually immediate. The major benefits for the electric system may arise from the deferral of grid expansion investments, decrease of technical losses and the reduction of customers' bills. However, some costs related to new systems to forecast critical periods, administrative costs of notification and technology development and implementation cost were assessed in the cost benefit analysis EDP Distribuição and INESC-TEC ran.

According to this, dynamic tariffs represent a new tool for DSOs to manage the grid. Together with consumption forecasting tools, dynamic tariffs allow DSOs to send a stronger price-signal, applicable to a short number of peak hours per year, after warning the customer in advance.

\section{DYNAMIC TARIFFS}

There are several types of dynamic tariffs, which can be selected according to the context and objectives of their implementation. EDP Distribuição has analyzed 4 types of schemes:

Critical Peak Pricing (CPP): Pre definition of higher prices to implement during critical hours, which can either reflect a higher price in the energy or a higher demand level for network capacity. Notification is usually made 1 to 2 days in advance and critical periods last for up to 8 hours;

$\circ \quad$ Real Time Pricing (RTP): Prices change on an hourly basis, usually linked to the prices in the wholesale market.

Peak Time Rebates (PTR) or Critical Peak Rebates (CPR): This scheme is similar to the CPP. However, instead of applying a higher price during critical hours, in this scheme a discount is given to customers that reduce their level of consumption in these hours;

○ Super peak ToU scheme: Under this static scheme, there is a set of hours where the customer receives a strong price-signal, in order to reduce consumption. This period can co-exist with a peak period.

EDP Distribuição is likely to implement in the pilots a CPP scheme due to its benefits: it's easy to be understood by customers; it provides a strong price signal that encourages the shift of consumption with great benefits for costumer's bills; the short duration of those periods. Also, this scheme has delivered the best results in terms of demand response, according to some literature (figure 2).

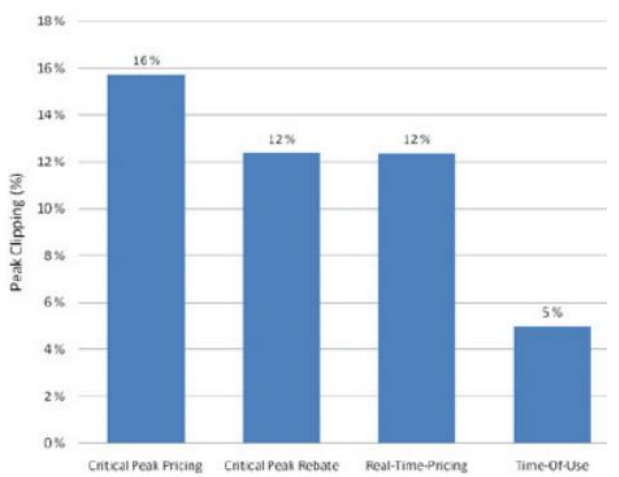

Figure 2 - Chart from the document "The potential of smart meter enabled programs to increase energy and systems efficienct: a mass pilot comparison", Vaasaett, 2011

However, there are some disadvantages that can't be neglected and may constitute a barrier for the success of such implementation such as the perception from clients regarding the price level or by being intrusive when a critical event occurs. Besides, a Super Peak ToU will also be tested in the pilots scheme, due to its potential benefits and lower implementation costs.

\section{THE PORTUGUESE TARIFF STRUCTURE}

In Portugal, the Network Access tariff structure for Very High Voltage (VHV), High Voltage (HV) and

Medium Voltage (MV) customers is Time-of-Use and comprises four different time periods: peak, full, off-peak and super off-peak. On the other hand, customers pay the Network Access through four different billing terms: contracted power (cp), average load in peak hours (alph), active energy (ae) and reactive energy (re). While monthy $\mathrm{cp}$ equals the customer's maximum load in the previous twelve months, average load in peak hours is only charged during peaks. The latter is the term from which network operators recover the majority of their revenues. From 2015 data, alph represented more than $40 \%$ of $\mathrm{HV}$ and more than half of all MV customers' spending with the Distribution Network charges. In fact, during peak hours it is frequent that Network Access costs more than the Energy component of the tariff paid by this segment of customers.

Economically, each of the four billing terms has a different goal. Contracted power is meant to recover peripheral network costs, average power during peaks shall recover the costs of investing in the central network assets, while energy is charged to recover the cost of energy losses in the different periods.

\section{EDPD Pilot}

The pilot project required by ERSE is expected to start in the second half of 2016. At least two tariff schemes are expected to be tested. One of them will be a Critical Peak Pricing scheme. This scheme will include a set of critical 
days for the network, each year. The number of critical days will be pre-set, and should be the duration of the critical period on those days. Also, the customer will be notified 1 or 2 days in advance, by email, message or in the internet, to ensure he is provided with the necessary time to adapt consumption during those events. The involved customers will also have access to a webportal where, besides being able to check their consumption, are able to simulate the benefits of responding to the pricesignals they are sent. It is important to highlight that the main billing term that will be changed in the new scheme will be aplh, which should be now applied to a much lower number of hours, but increase its value, to make the response more effective.

The other scheme to be analysed will be Time-of-Use, and new load terms and prices shall be introduced. Even though some parameters of the new schemes are still to be specified, there are two main goals to reach: 1) to better match peak prices to the hours where the grid tends to be most congested; 2) to lower the number of peak hours from the current 1.000 to approximately 100 .hours. By doing this, the price-signal applied to the customers during critical hours will be higher than it is now, but they will pay a lower price in the remaining hours. On average, however, it is expected that revenues coming from $\mathrm{HV}$ and MV do not change if they keep their consumption profile the same. Their savings should be related to their demand response and load shifts.

Another important issue has to do with the definitions of critical day and network congestion. An approach that considers unilateral energy flows from generation points to consumption will define a critical day as a day when there is a very high level of energy flowing through the grids. For instance, a congestion at a HV level would correspond to a moment when the summation of LV, MV and HV reaches its highest values. That is, its congestion function would be the following:

$$
\text { Congestion }_{H V}=\sum C_{H V}+C_{M V}+C_{U V}
$$

A different approach takes distributed generation into account, and considers congestion as a period when there is a high level of consumption net of distributed generation. Under this approach, price-signals would be stronger when more consumption is satisfied by nondistributed generation. This also means that the new scheme would tend to maximize the use of renewable energy sourced (RES). Hence, and using the HV network example again, a congestion at the HV level would be calculated through the following equation:

$$
\begin{aligned}
\text { Congestion }_{H V}= & \sum_{-R E S_{M V}} C_{H V}+C_{M V}+C_{U V}-R E S_{U V} \\
& =S_{H V}
\end{aligned}
$$

The VHV, HV and MV customers adopting these schemes will not necessarily be charged a higher price in the periods when they consume more, because the critical hours at the network level are highly influenced by the consumption of Low Voltage customers. This is why the critical hours of network congestion, for both approaches, tend to be concentrated in the Winter months, and occur mostly between 6PM and 10PM.

Under the Portuguese Tariff Code, there is the principle of tariff uniformity. This means that customers of the same nature shall be charged the same for the services provided in the whole country. Despite this principle, it may become possible that the pilot will contemplate tariff schemes with the exact same rules - same number of critical days per year, same types of prices - but a regional application of critical days. This would be done because of the regional nature of network congestions. While an analysis of consumption net of RES in the whole country shows that Winter months are the most critical, if we look at the South of Portugal we realize some of the critical hours in this region occur in the Summer, due to the touristic sector.

The pilot will also contemplate customer engagement. For this purpose, a webportal will be created. HV and MV customers already have access to their consumption history. However, this webportal will allow them to compare what they pay for under the new and the former schemes. Also, they will be able to simulate how much they can save if they reduce or shift consumption from critical to non-critical hours.

This pilot is expected to provide some results about potential benefits and costs of adopting dynamic tariffs for Network Access. A set of Key Performance Indicators (KPIs) will also be chosen, to better quantify the pilot's success. These KPIs must take into account variables like total consumption, consumption in critical periods, consumer savings and peak consumption growth. INESCTEC and EDP Distribuição will also report the evolution of the pilot project through a set of periodical monitoring reports.

\section{THE COST-BENEFIT ANALYSIS}

As mentioned above, the Portuguese Energy Services Regulatory Agency, ERSE, required in 2014 that EDP Distribuição submitted a Plan to develop Pilot Projects towards the implementation of Dynamic Tariffs regarding the access to the networks. This Plan should include details about the pilot projects as well as the results of a Cost-Benefit Analysis, CBA. Despite this requirement is directed to a DSO, it was clear from the beginning that the CBA should investigate the benefits and the costs for the entire Portuguese power system. Having this in mind, the CBA addressed the following points:

Benefits from load shifting considering the market Social Welfare Function, SWF, taking into 
account the participation of Portugal in Iberian wholesale market;

The reduction of network losses;

Investment deferral;

Cost reduction associated to the procurement of secondary and terciary reserves;

The costs of implementing the pilot projects as well as the costs during the horizon under analysis.

The evaluation was conducted taking the 100 hours of larger demand in 2014 as a departing point. Regarding the impact on the SWF, for each critical hour $h$ it was evaluated the reduction of the value of SWF due to the reduction of $5 \%$ of the demand in EHV, $\mathrm{HV}$ and $\mathrm{MV}$ levels (approximately corresponding to $50 \%$ of the total Portuguese demand) in that hour. Then, it was also evaluated how the SWF of the hours $h-1$ and $h+1$ increased due to the increase of the demand. In this analysis we admitted that the 5\% demand reduction on hour $h$ was equally distributed by hours $h-1$ and $h+1$. There would exist a benefit if the increase of SWF in hours $\mathrm{h}-1$ and $\mathrm{h}+1$ was larger than reduction in hour $\mathrm{h}$. This analysis was conducted to the 100 hours of larger demand in 2014 and the results were then projected along the horizon under analysis from 2015 to 2030.

Regarding the network losses, we used the historical demand data for 2014, and loss profiles provided by ERSE. These loss profiles are calculated on a 15 min basis and for each voltage level and type of client they relate the demand and the losses in the system. Therefore, the losses were estimated on the departing point and then once again admitting that $5 \%$ of the demand on EHV, HV and MV on the 100 hours of larger demand was transferred to the lateral hours. The difference among these two situations once again projected till 2030 corresponds to the benefit coming from this item.

In what concerns the network investments, it should be clarified that currently investment decisions are taken by the DSO based in the evolution of the peak demand, that is, guarantying a worst case scenario. This means that if a reduction of the peak demand is induced by the adoption of dynamic tariffs then some investments in network assets can be avoided or at least deferred. Accordingly, we used the utilization factors of network equipment (percentage of maximum flow in an equipment regarding its capacity) for 2014 and projected these values till 2030 taking into account the expected demand evolution. The investment requirements were estimated adopting an investment trigger of $75 \%$ and admitting that the commissioning of the new equipment occurred 5 years afterwards. This analysis conducted to a reference investment level. Then, the analysis was repeated considering that the demand of the EHV, HV and MV levels on the 100 hours of larger demand reduced by $5 \%$ thus originating a peak demand reduction. The new investment requirement was compared with the reference value leading to the corresponding potential benefit.

The impact on contracting reserves, namely secondary and tertiary reserves, was also investigated but it was found to be neglectable. In fact, secondary bid prices were found to be very close (sometimes with differences of few cents of $€)$ so that interchanging the amount of contracted reserve from one hour to other hours would virtually have no economic impact. Additionally, the amount of reserves to contract bu the TSO also depend on the amount of wind and photovoltaic generation estimated for each hour of the next day. This suggests that even if some demand reduction is induced in some hours by dynamic tariffs, the amount of reserves to be contracted by the TSO would remained almost unchanged.

Finally, EDP Distribuição identified costs related to the development of forecasting models for demand, distributed generation and critical hours on a regional and a voltage level basis, with programs to increase the awareness of customers, with communicating with consumers and with the adaptation of the billing software to consider the new tariff options.

The results obtained so far suggest a net benefit of around $7 \mathrm{M} €$, taking into account a discount rate of $6,75 \%$. It should also be mentioned that largest share of the potential benefits, around $98 \%$, is related to network investment deferral over the horizon till 2030 and that the benefits associated with the impact on the SWF, on the networks losses and on contracting reserves were found marginal.

\section{MAIN CONCLUSIONS}

The pilot project of dynamic tariffs for network access can be seen as pioneer experiment of testing dynamic tariffs on the network component. The participation of all stakeholders - suppliers, consumers and energy services companies - is key to make sure the pilot is successfully implemented. There should be adequate indicators to assess how dynamic tariffs can change aggregate and peak consumption. Also, the total benefits of these new schemes should not only be seen as DSO or customer benefits: all System gains and costs shall be taken into account during the pilot. Although some of the expected gains are longterm-related, the pilot should help understand how much impact this type of tariffs will have on customer behavior and on network costs. In case there are clear net benefits associated to this pilot, the Portuguese NRA will take the results of the pilot into account to eventually include this tariff option for $\mathrm{VHV}, \mathrm{HV}$ and $\mathrm{MV}$ customers in the future.

\section{REFERENCES}

[1] - ERSE - Regulamento Tarifário do Setor Elétrico, 2014.

[2] - Vaasaett, 2011 - Empower demand

[3] - Eurelectric, 2013 - Network tariff structure for a Smart Energy System

[4] - EDP Distribuição 